\title{
The Long-Term Fate and Toxicity of PEG-Modified Single-Walled Carbon Nanotube Isoliquiritigenin Delivery Vehicles in Rats
}

\author{
Bo Han, ${ }^{1,2}$ Mingming Zhang, ${ }^{1}$ Ting Tang, ${ }^{3}$ Qiusheng Zheng, ${ }^{1,2}$ \\ Keyu Wang, ${ }^{1}$ Le Li, ${ }^{1,2}$ and Wen Chen ${ }^{1,2}$ \\ ${ }^{1}$ Pharmacy School of Shihezi University, Shihezi 832002, China \\ ${ }^{2}$ Key Laboratory of Xinjiang Phytomedicine Resources, Shihezi 832002, China \\ ${ }^{3}$ The Ankang Hospital of Xinjiang P\&C Corps, Shihezi 832002, China
}

Correspondence should be addressed to Wen Chen; chen-wen2000@126.com

Received 28 October 2013; Revised 11 December 2013; Accepted 12 December 2013; Published 16 March 2014

Academic Editor: Jinlong Jiang

Copyright $\odot 2014$ Bo Han et al. This is an open access article distributed under the Creative Commons Attribution License, which permits unrestricted use, distribution, and reproduction in any medium, provided the original work is properly cited.

Oxidized single-walled carbon nanotubes (o-SWNTs) was modified by covalently and noncovalently linking PEG to the o-SWNTs. The influence of oxidation time, PEG molecular weight, and type of PEG linkage on the blood clearance time of PEG-modified single-walled carbon nanotubes (SWNTs) was investigated. The toxicity profile of SWNTs covalently linked to PEG (c-PEG-oSWNTs) in rats has also been determined. The pharmacokinetics of c-PEG-o-SWNTs in rats and their distribution in vital organs were monitored by Raman spectroscopy, and the blood clearance of homogenate isoliquiritigenin (ISL) was determined by HPLC. Photos of tissue and tissue sections were taken to evaluate the toxicity of c-PEG-o-SWNTs. We found that SWNTs which were covalently modified with PEG and have a molecular weight of 3500 had the longest blood clearance half-lives. However, SWNTs were toxic to the kidneys and the hearts. The high renal clearance of long-term fate SWNTs may occur because of impaired kidney filtration function. Therefore, we assume that while researchers study the long-term fate of SWNTs, the toxicity of SWNTs also needs to be taken into account.

\section{Introduction}

The root of Glycyrrhiza uralensis Fischer is widely used as a harmonizing ingredient in traditional herbal formulations in oriental medicine; it is used in more formulations than any other herb. One of the most notable ingredients in Glycyrrhiza uralensis Fischer is isoliquiritigenin (ISL), a simple chalcone-type flavonoid that has antioxidative effect $[1,2]$, antiplatelet aggregation effect [3], anti-ischemia effect [4], anti-inflammatory property $[5,6]$, antispasmodic effect [7], and estrogenic property [8]. In the past seven years, our group has expanded the study of ISL drug performance [9-11] and has made progress in ISL tumor pharmacology [12], natural product chemistry [11], and in vivo analysis [13]. In this study, we aim to improve the target performance of ISL by delivering the compound with the help of SWNTs.
SWNTs have been heavily studied because of their unique physicochemical properties and potential applications [14]. Encouraged by the success of using carbon nanotubes (CNTs) for in vitro sensing, drug delivery, and imaging, researchers in this field have moved to in vivo investigations in animals [15].

In vivo biodistribution andpharmacokinetic studies have been carried out bya number of groups using different CNT materials. Many of these studies confirmed the poor biocompatibility of original carbon nanotube formulations [16]. Purified SWNTs implanted in mice via intratracheal instillation resulted in epithelioid granulomas and interstitial inflammation in a 90-day study [17]. Lam et al. found that undoped multiwalled CNTs (MWCNTs) can induce severe granulomatous inflammatory responses compared with nitrogen-doped MWCNTs when administered intratracheally in mice [18]. Original MWCNTs inhaled by mice 
for $6 \mathrm{~h}$ were found throughout the lungs and significant pulmonary toxicity was observed [19].

SWNTs used for drug delivery are usually functionalized with polyethylene glycol (PEG). PEG provides a desirable coating for nanotubes and nanoparticles, because it reduces their immunogenicity and reduces their chance of being nonspecifically taken up by cells of the reticuloendothelial system. Consequently, the phagocytosis of nanoparticles is diminished and their clearance time is prolonged when modified with PEG [20].

The purpose of our study is to investigate the influence of SWNTs' oxidation time, PEG molecular weight, and PEG linkage type on the clearance time of SWNTs. After the best long-term fate single-walled carbon nanotubes (LTFS) were synthesized, the blood clearance time changes between ISL and ISL loaded with LTFS were investigated. The toxicity of LTFS in rats was also studied. While there are many researchers concern about the toxicity of carbon nanotube as well as its long-term fate, there is little work focusing on the relationship between the toxicity and the long-term fate of CNTs.

\section{Materials and Methods}

\subsection{Reagents, Instruments, and Test Animals}

2.1.1. Reagents. SWNTs were obtained from Nanjing Jicang Technology Co., Ltd (purity $\geqslant 90 \%$ ); ISL was purchased from Tianye Chemical Co., Ltd. (purity $\geqslant 98 \%$ ); NHydroxysuccinimide (NHS) and carbodiimide $\mathrm{HCl}$ (EDC . $\mathrm{HCl}$, purity $\geqslant 98.5 \%$ ) were obtained from GL Biochem (Shanghai), Ltd.; $\mathrm{NH}_{2}-\mathrm{PEG}-\mathrm{NH}_{2}$ (MWs of 2000, 3500, and 5000) were purchased from Beijing Kaizheng Biotech Development Co., Ltd. Heparin sodium injection was purchased from Tianjin Biochemical Co., Ltd.; mixed phosphate buffer solution (PBS) was purchased from Sigma-Aldrich; and nitric acid, sulfuric acid, ethyl acetate, dimethyl sulfoxide, and anhydrous ethanol (A.R.) were purchased from Sinopharm Chemical Reagent Co., Ltd.

2.1.2. Instruments. An STA409PC composite thermal analyzer and $\mathrm{Al}_{2} \mathrm{O}_{3}$ crucible were used in this study (NETZSCH Scientific Instrument Trading (Shanghai) Co., Ltd.). An H600 transmission electron microscope (Japan Electronics Co., Ltd.); a Raman spectrometer (Bruker 70), a dialysis bag (MWCO 7000-14000; Hainan Nanjing Science and Technology Development Co., Ltd.), an LC-20A high-performance liquid chromatograph (Shimadzu); and a chromatographic column (Symmetry 300, $\mathrm{C}_{18}, 5 \mu \mathrm{m}, \Phi 4.6 \times 150 \mathrm{~mm}$ ) were also used.

2.1.3. Test Animals. Six-week-old Wistar rats with body weight of $200 \pm 20 \mathrm{~g}$ and production certificate number of SCXK (Xin)-2003-0001 (Animal Center of Xinjiang Medical University) were used in this study.

2.2. Preparation of PEGylated Nanotubes. One hundred $\mathrm{mg}$ of $\mathrm{HiPco}$ SWNTs were added to $50 \mathrm{~mL}$ of a $1: 1 \mathrm{HNO}_{3}-\mathrm{H}_{2} \mathrm{SO}_{4}$ solution. The mixture was refluxed for $15 \mathrm{~min}, 30 \mathrm{~min}$, or $45 \mathrm{~min}$ at $140^{\circ} \mathrm{C}$, respectively, and then cooled. The mixture was diluted with $250 \mathrm{~mL}$ of deionized water and filtered through a microporous membrane. The filter film containing oxidized SWNTs (o-SWNTs) was then washed with deionized water. The carboxyl groups present on o-SWNTs surfaces provide the binding sites required for attaching PEG.

Covalently linked PEG to o-SWNTs (c-PEG-o-SWNTs) was prepared according to the following steps. $50 \mathrm{mg}$ of oSWNTs was combined with $50.00 \mathrm{~mL}$ of phosphate buffer ( $\mathrm{pH}$ 6.8) under sonication to achieve an evenly dispersed solution. $200 \mathrm{mg}$ carbodiimide (EDC) and $250 \mathrm{mg}$ NHS were added into the dispersed solution and then the mixture was sonicated for $30 \mathrm{~min}$. After that, $50 \mathrm{mg} \mathrm{NH} \mathrm{N}_{2}-\mathrm{PEG}-\mathrm{NH}_{2}$ (MW of 2000,3500 , or 5000) was added and the mixture was magnetically stirred at room temperature for $24 \mathrm{~h}$. Distilled water was added to achieve a final volume 3 times larger than initial volume. The diluted mixture was centrifuged at $22000 \mathrm{~g}$ for $1 \mathrm{~h}$. The supernatant was placed in a dialysis bag dialysed to neutral $\mathrm{pH}$ [21]. The amount of grafted PEG was determined by the thermal gravimetric analysis method (TG) [22].

Noncovalently linked PEG to o-SWNTs (n-PEG-oSWNTs) was prepared according to the following steps. $50 \mathrm{mg}$ of o-SWNTs was combined with $50.00 \mathrm{~mL}$ of $\mathrm{pH} 6.8$ phosphate buffer solution and then sonicated for $30 \mathrm{~min}$. The solution obtained was combined with $50 \mathrm{mg} \mathrm{NH}$-PEG- $\mathrm{NH}_{2}$ (MW 2000) and stirred evenly at room temperature for $24 \mathrm{~h}$ to get n-PEG-o-SWNTs [21]. The amount of grafted PEG was determined by TG [22].

2.3. Preparation of ISL and Functionalization of SWNTs Complexes. In order to attach ISL onto c-PEG-o-SWNTs noncovalently, $12 \mathrm{mg}$ of ISL was dissolved in a mixture of DMSO, PEG400, and saline $(1: 2: 9)$ to make a $1 \mathrm{mg} / \mathrm{mL}$ ISL solution. $60 \mathrm{mg}$ of c-PEG-o-SWNTs was added to the ISL solution and the mixture was stirred for $6 \mathrm{~h}$ in a $25^{\circ} \mathrm{C}$ water bath to obtain SWNT-ISL complexes (c-PEG-o-SWNTsISL). The prepared products were resuspended and stored at $4^{\circ} \mathrm{C}$.

2.4. Animal Experiments. Twenty-four hours after injecting $200 \mu \mathrm{L}$ of the c-PEG-o-SWNTs-ISL test solution into rats' tail veins, $0.5 \mathrm{~mL}$ of blood was obtained through orbital veins and placed into heparin sodium treated centrifuge tubes. After centrifuging the blood for $10 \mathrm{~min}$ at $5000 \mathrm{rpm}, 200 \mu \mathrm{L}$ plasma was collected for future use. Then rats were killed by cervical dislocation, and their hearts, livers, spleens, lungs, kidneys, and brains were collected and weighed. The various tissues and organs were placed in $4 \mathrm{~mL}$ centrifuge tubes containing $2 \mathrm{~mL}$ of normal saline and homogenized. Two portions of homogenate $(7.5 \mathrm{~mL})$ were taken. One portion was used to determine the ISL content by HPLC. The other portion was combined with $0.75 \mathrm{~mL}$ of CMC-Na solution and then homogenized by vortex oscillation for $5 \mathrm{~min}$. This portion was reserved for Raman spectroscopy. 
2.5. Raman Measurement of PEG-SWNT Solutions. Various concentrations of SWNT solutions were drawn into capillary glass tubes (Fisher) and measured using a Bruker 70 Raman spectrometer (laser excitation wavelength was $1024 \mathrm{~nm} ; 30 \mathrm{~min} ; 850$ times). Four spectra were measured for each sample to obtain an average spectrum. For a given concentration of SWNTs, the Raman intensity was obtained by integrating the PEG-o-SWNT G-band peak area from $1570 \mathrm{~cm}^{-1}$ to $1620 \mathrm{~cm}^{-1}$. The Raman G-band peak areas were used to calculate PEG-o-SWNT concentrations in the blood. The percentage of injected dose per gram (\%ID/g) of blood was calculated using the following equation:

$$
\begin{aligned}
& \% \mathrm{ID} / \mathrm{g} \\
& =\frac{[\mathrm{SWNTs}]_{\text {blood lysate }} \times V_{\text {blood lysate }}}{[\mathrm{SWNTs}]_{\text {injected }} \times V_{\text {injected SWNTs }} \times \text { tissue weight }} \times 100 \% \text {. }
\end{aligned}
$$

2.6. Determination of Isoliquiritigenin Content in the Tissue Homogenate with HPLC. The mobile phase consisted of a mixture of acetonitrile and double distilled water $(1: 1(\mathrm{v} / \mathrm{v})$, the flow rate was $10 \mathrm{~mL} / \mathrm{min}$, and the column temperature was $40^{\circ} \mathrm{C}$. A sample volume of $20 \mu \mathrm{L}$ was injected into the HPLC. The ISL detection wavelength was $368 \mathrm{~nm}$ and the operating time was $9 \mathrm{~min}$.

2.7. Statistical Analysis. The pharmacokinetic parameters were performed by DAS (ver1.0) program and compared by statistical analysis. One-way ANOVA was used for parametric data, when differences were statistically significant $(P<$ 0.05).

\section{Results and Discussion}

3.1. Characterization of PEG-Modified SWNTs. The structure of c-PEG-o-SWNTs after synthesized by EDC is shown in Figure 1. SWNTs covalently functionalized by PEG can form stable solution in water [23]. Centrifugation was used to remove big bundles and impurities, leaving short individual and small bundles of tubes in solution. Strong resonance Raman scattering is an intrinsic optical property of SWNTs, with sharp peaks and low background in the spectra. The tangential graphite-like phonon mode ( $\mathrm{G}$ band), which is the strongest peak in the SWNTs Raman spectrum, was used to detect nanotubes in blood and tissue lysates.

3.2. Determination of c-PEG-o-SWNTs In Vivo by Raman Spectroscopy. SWNTs have a unique crystal structure, which produces unique Raman scattering at a wavelength of about $1590 \mathrm{~nm}$. SWNTs retain the feature of Raman scattering after covalent or noncovalent modification with PEG. Figure 2 shows the Raman spectra of different concentrations of cPEG-o-SWNTs in plasma solutions. The G bands of SWNTs were absorbed, and the absorption strength increased as the c-PEG-o-SWNT concentration in solution increased. With the above positive correlation, c-PEG-o-SWNTs in the blood and tissue fluid were quantified by using the peak area of the

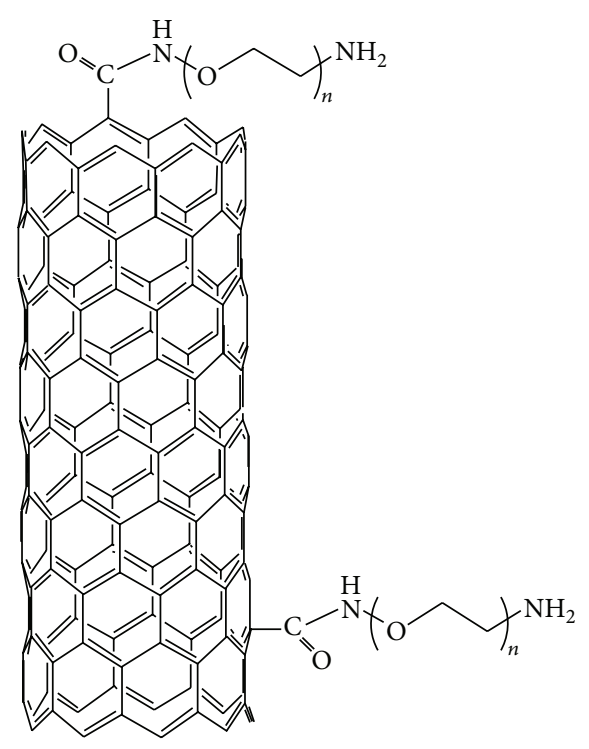

FIGURE 1: The structure of c-PEG-o-SWNTs.

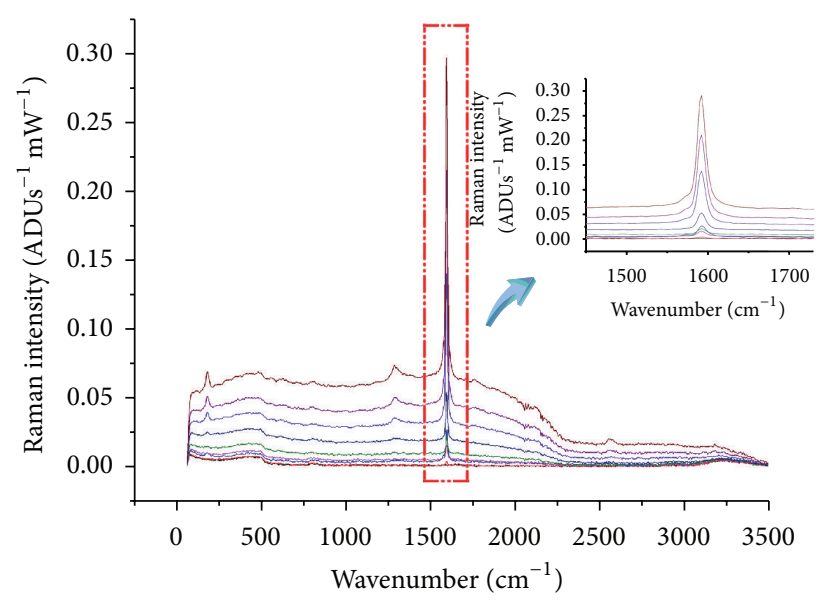

FIGURE 2: The Raman spectra of different concentrations of c-PEGo-SWNTs in blood samples.

$\mathrm{G}$ band. The data were fitted by the nonlinear standard curve $y=0.0164 X-2.123 \times 10^{-5} X^{2}-0.07973(R=0.9935)$ using Origin 7.5 software. The results of the sampling recovery test, precision test, and durability test showed that the amount of c-PEG-o-SWNTs in the animals' tissues and blood were accurately determined by using this method.

3.3. Determination of Isoliquiritigenin Content in Rat Tissues by HPLC. The ultraviolet absorption spectrum of ISL with a maximum absorption wavelength of $368 \mathrm{~nm}$. ISL was eluted at $5.1 \mathrm{~min}$ under the HPLC conditions employed in this work, while blank plasma revealed no peak at or around $5.1 \mathrm{~min}$ under the same conditions, suggesting the good specificity of determining ISL by HPLC. The results of the sampling recovery test, precision test, and durability test displayed that the ISL content in the animals' tissues and blood were accurately assayed by this method [11]. 


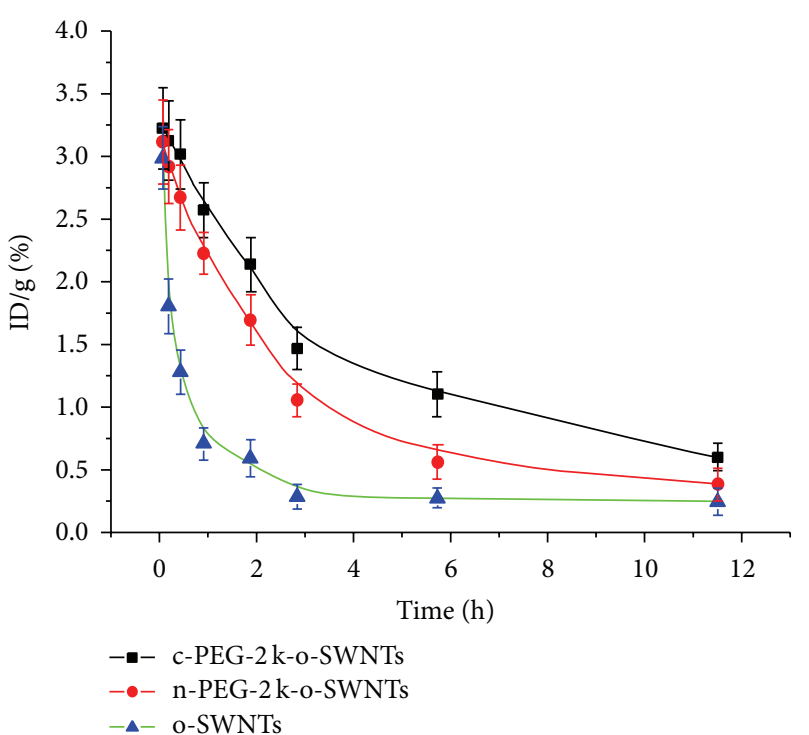

(a)

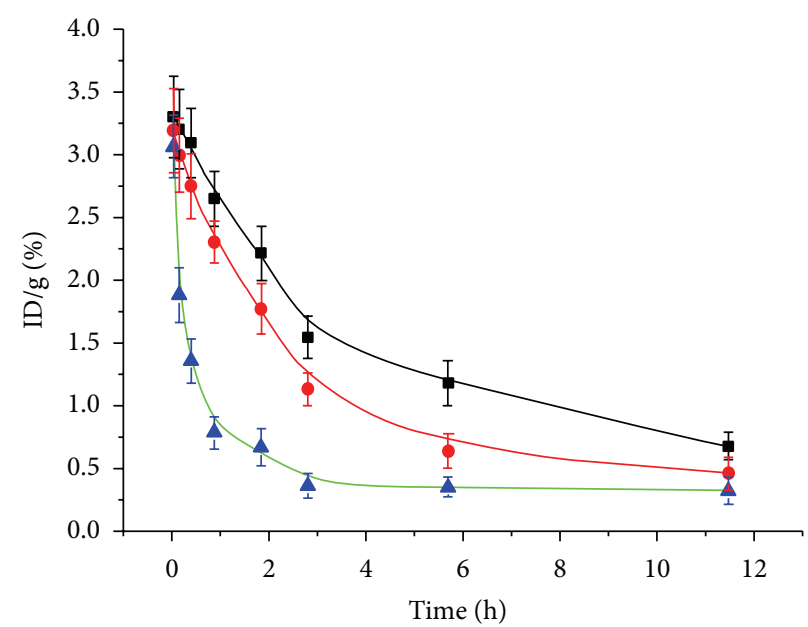

- 15 min-PEG-2 k-o-SWNTs

- -30 min-PEG-2 k-o-SWNTs

-₫- 45 min-PEG-2 k-o-SWNTs

(b)

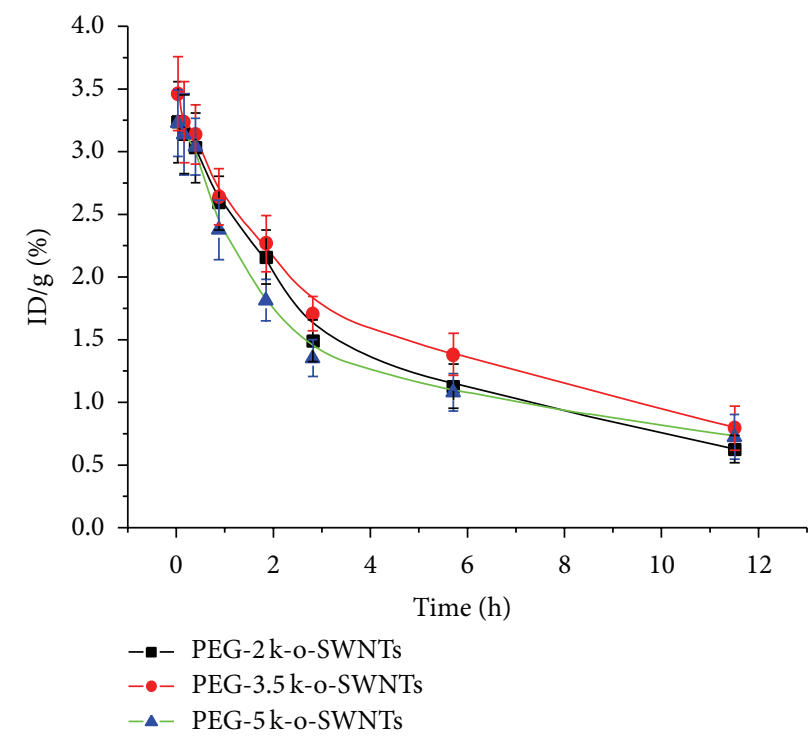

(c)

FIGURE 3: The investigation of the influence of different types of PEG modification factors (a), different degrees of oxidation on SWNT clearance time (b), and the influence modifying SWNTs with different PEG molecular weights (c) on the SWNT clearance time in vivo.

3.4. Investigation on Factors That Influence the Blood Clearance Time of SWNTs. Figure 3(a) shows the influence of different types of PEG modification (covalent, noncovalent, or no PEG modification) on the blood clearance time of SWNTs. The half-lives of SWNTs were $t_{1 / 2(\mathrm{o}-\mathrm{SWNTs})}=0.24 \mathrm{~h}$, $t_{1 / 2 \text { (c-PEG-2 k-o-SWNTs) }}=2.35 \mathrm{~h}$, and $t_{1 / 2 \text { (n-PEG- } 2 \mathrm{k} \text {-o-SWNTs) }}=$ 1.75 h. Covalently linking $\mathrm{NH}_{2}-\mathrm{PEG}-\mathrm{NH}_{2}$ (MW 2000) significantly prolonged the clearance time of SWNTs, but noncovalently linking $\mathrm{NH}_{2}-\mathrm{PEG}-\mathrm{NH}_{2}$ (MW 2000) to SWNTs did not. These results imply that noncovalent modification of SWNTs using $\mathrm{NH}_{2}-\mathrm{PEG}-\mathrm{NH}_{2}$ (MW 2000) may not protect SWNTs from clearance by the reticuloendothelial system. In contrast, covalently linking $\mathrm{NH}_{2}-\mathrm{PEG}-\mathrm{NH}_{2}$ (MW 2000) increases the blood clearance time of SWNTs.
Figure 3(b) shows the influence of different degrees of oxidation on SWNT clearance time. Using TG calculation, the carboxyl contents were found to be $9.44 \%, 13.18 \%$, and $15.32 \%$ at $15 \mathrm{~min}, 30 \mathrm{~min}$, and $45 \mathrm{~min}$ of oxidation, respectively. Through the DAS 1.0 pharmacokinetic software calculation, we found that CNTs covalently linked to $\mathrm{NH}_{2}$ PEG-NH $\mathrm{NH}_{2}$ (MW 2000) caused blood clearance half-lives of $1.57 \mathrm{~h}, 2.35 \mathrm{~h}$, and $1.61 \mathrm{~h}$ according to oxidation time of $15 \mathrm{~min}$, $30 \mathrm{~min}$, and $45 \mathrm{~min}$, respectively. These results suggest that 30 min of mixed acid oxidation results in SWNTs that have the longest blood clearance time. Oxidation time longer than 30 min did not extend the clearance time of SWNTs.

Figure 3(c) shows the influence of modifying SWNTs with different PEG molecular weights on the SWNT 


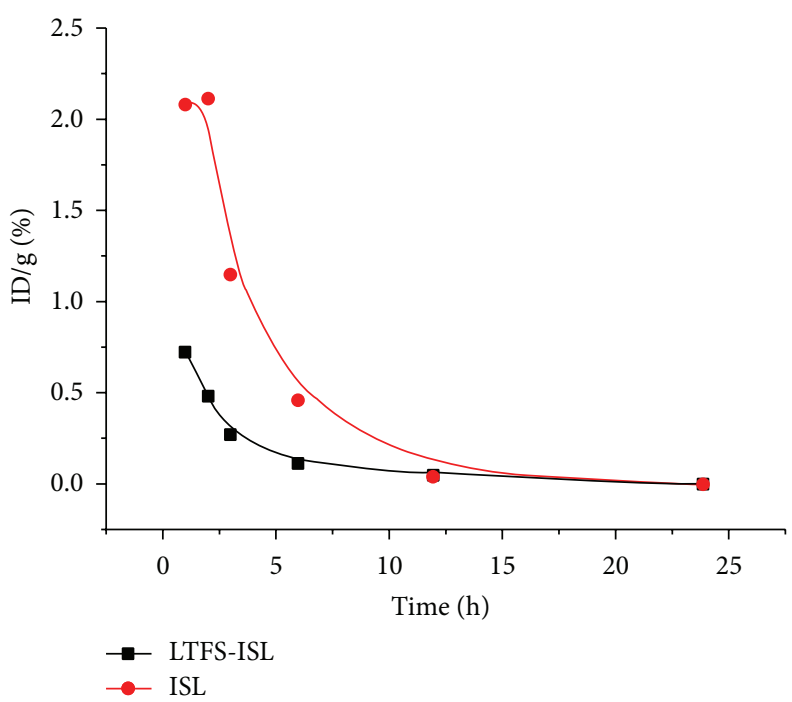

FIGURE 4: The blood concentration of ISL with and without LTFS. The $y$-axis represents injected dose per gram (\%ID/g).

clearance time. Through calculation, the half-lives were $t_{1 / 2(\mathrm{c}-\text { PEG-2 } \mathrm{k}-\mathrm{o}-\mathrm{SWNTS})}=2.35 \mathrm{~h}, t_{1 / 2(\mathrm{c}-\mathrm{PEG}-3.5 \mathrm{k} \text {-o-SWNTs })}=2.77 \mathrm{~h}$, and $t_{1 / 2(\mathrm{c}-\mathrm{PEG}-5 \mathrm{k}-\mathrm{o}-\mathrm{SWNTS})}=2.41 \mathrm{~h}$. Although PEG modification on the surface of SWNTs prolonged their blood clearance time, the molecular weight of $\mathrm{NH}_{2}-\mathrm{PEG}-\mathrm{NH}_{2}$ that can prolong blood clearance time to the highest level remains unclear.

In summary, these results suggest that $30 \mathrm{~min}$ of mixed acid oxidation of SWNTs followed by covalently linking $\mathrm{NH}_{2}$ PEG- $\mathrm{NH}_{2}$ (MW 3500) resulted in SWNTs with the longest blood clearance time. After the formulation of the best LTFS was obtained, ISL was incorporated into the LTFS (LTFSISL), and the pharmacokinetic profile and the toxicity of LTFS-ISL on rats were determined.

The research group led by Dai et al. at Stanford University found that ultrasonic dispersion of multiwalled carbon nanotubes with a molecular weight of 7000 for $1 \mathrm{~h}$ resulted in a long-term fate of one day after tail vein injection [16]. However, the Hudson group at the University of New Mexico has contended that one hour of ultrasonic dispersion will break the long chain of PEG and thereby reduce the actual molecular weight of the PEG on the carbon nanotubes [24]. In this work, we did not use ultrasonic-assisted dispersion of c-PEG-o-SWNTs, and our results suggest that SWNTs covalently linked to PEG with a molecular weight of 3500 display the longest blood clearance half-live.

3.5. Influence of LTFS on ISL Blood Clearance Time and Distribution. Figure 4 shows the concentration of ISL in blood with or without the SWNT carriers. Using the DAS 1.0 pharmacokinetic software, we determined that $t_{1 / 2 \text { ISL }}=$ $2.51 \mathrm{~h}$ and $t_{1 / 2 \text { (LTFS-ISL) }}=0.95 \mathrm{~h}$, demonstrating that the existence of carriers reduces the half-life of ISL in the blood.

Interestingly, as shown in Figure 4, the existence of carriers significantly reduces the concentration of ISL in blood. Thirty minutes after LTFS-ISL was injected through tail veins, the rats developed gross hematuria and black discharge in the urine, raising the possibility that LTFS-ISL may cause renal toxicity and that carbon nanotubes were discharged through the urine.

3.6. Appearance Changes in the Heart and Kidneys after the Injection of LTFS-ISL. We compared the hearts, livers, spleens, lungs, and kidneys of rats injected with ISL and LTFS-ISL. The results showed that the hearts and kidneys of rats in the LTFS-ISL group were blacker and larger than those of rats in the ISL group, suggesting that the carbon nanotubes remained in the hearts and kidneys, as shown in Figure 5.

3.7. Tissue Distribution of c-PEG-3.5 k-o-SWNTs-30 min. We determined the content of carbon nanotubes in the tissues (hearts, livers, spleens, and kidneys) and in the urine of rats injected with LTFS using Raman spectroscopy. Figure 6 shows the content of LTFS in the tissues and urine. It was discovered that the content of LTFS in the urine, liver, spleen, and kidney was higher than that in other organs. The results suggest that, like most nanosubstances, macrophages readily consume LTFS in the liver, spleen, and other organs through phagocytosis.

It should be noted that the discharged urine contained a large amount of carbon nanotubes, which is consistent with reports by Singh et al. [25] and Lacerda et al. [26], indicating that most carbon nanotubes are removed through kidney. From our experimental results and the work presented by Singh and Lacerda, we speculate that ISL can be adsorbed onto the surface of LTFS. Therefore, after LTFS are removed by kidney and the reticuloendothelial system, the concentration of ISL in blood significantly decreases.

3.8. Histopathology Changes after Injecting LTFS-ISL in the Heart and Kidney Tissues. We examined the heart and kidney tissue sections after H\&E staining. As illustrated in Figure 7, the myocardial cell cytoplasm of LTFS-ISLtreated rats changed into granules and vacuoles in some 

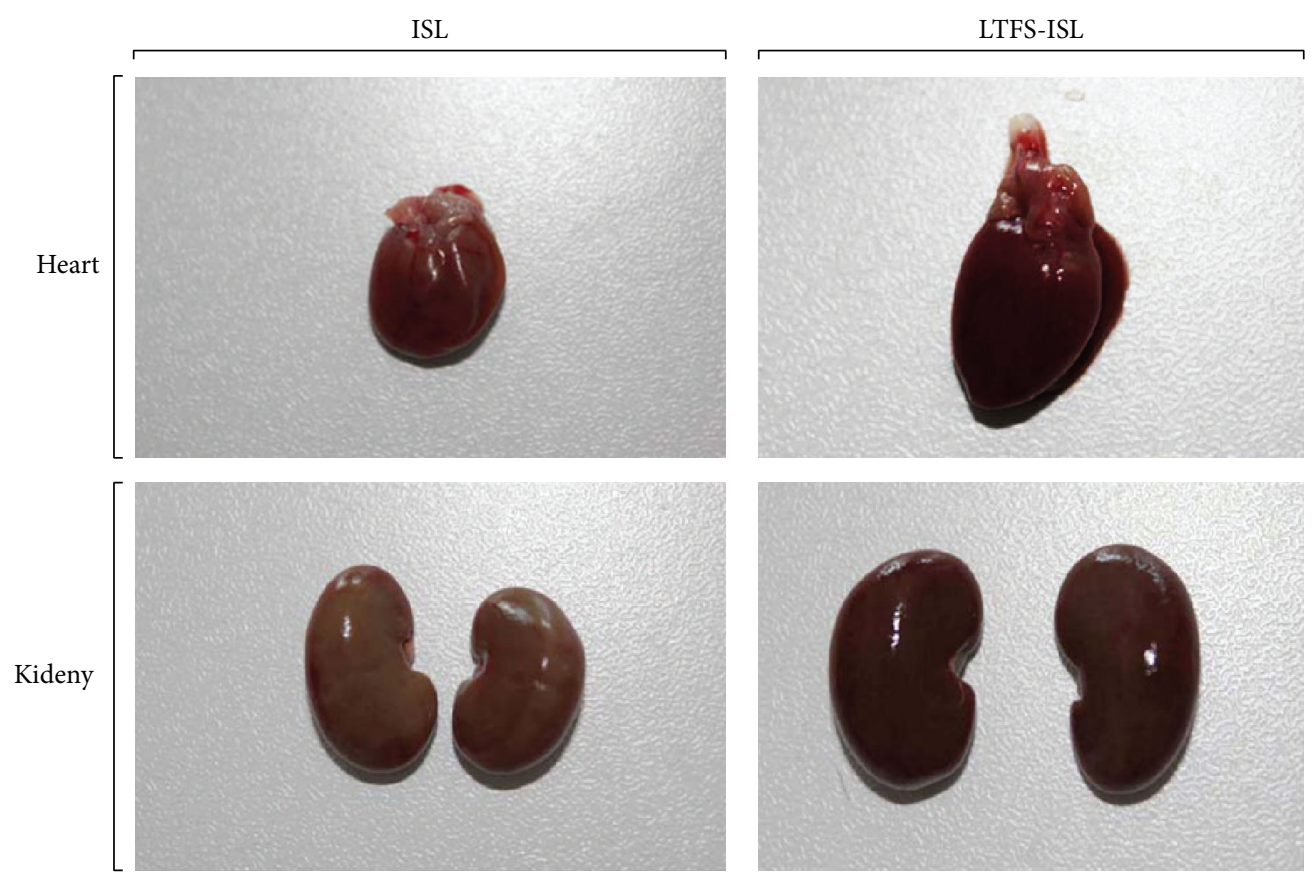

FIGURE 5: The influence of ISL and LTFS-ISL on the appearance of various organs.

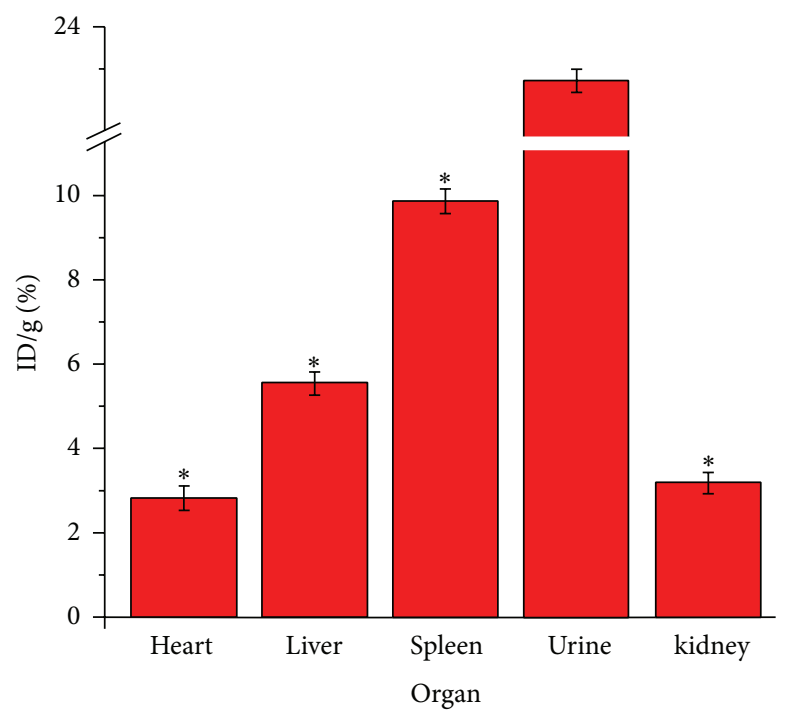

FIGURE 6: The distribution of LTFS $(n=6)$ in the tissues and urine. “*” indicates the differences were statistically significant compared with urine $\operatorname{bar}(P<0.05)$.

regions, and the color of the myocardial fibers faded and the nuclei of myocytes shrank. In addition, there were vacuolar deformations and particulate deformations in kidney tubules after LTFS-ISL administration. The above results suggest that LTFS-ISL may cause heart and kidney toxicity. The large discharge of LTFS-ISL in urine may be attributed to glomerular damage caused by LTFS-ISL.

\section{Conclusions}

We speculate that LTFS-ISL is toxic to kidney and heart. The results of our study showed that the longest half-life of PEG-o-SWNTs was obtained after SWNTs underwent reflux oxidation for $30 \mathrm{~min}$ and were covalently linked with $\mathrm{NH}_{2}-\mathrm{PEG}-\mathrm{NH}_{2}$ (MW 3500). These results indicate that surface modification of SWNTs with $\mathrm{NH}_{2}$-PEG- $\mathrm{NH}_{2}$ indeed prolongs their clearance time, and c-PEG-o-SWNT carriers reduce the blood clearance half-life of ISL. The black and red discharge in urine and postmortem tissue sections infer that LTFS are likely to cause liver and kidney toxicity. The increased renal clearance of LTFS may occur owing to impaired kidney filtration function. This discovery provides a new way to explain of long-term fate SWNTs' high renal clearance in rats. 

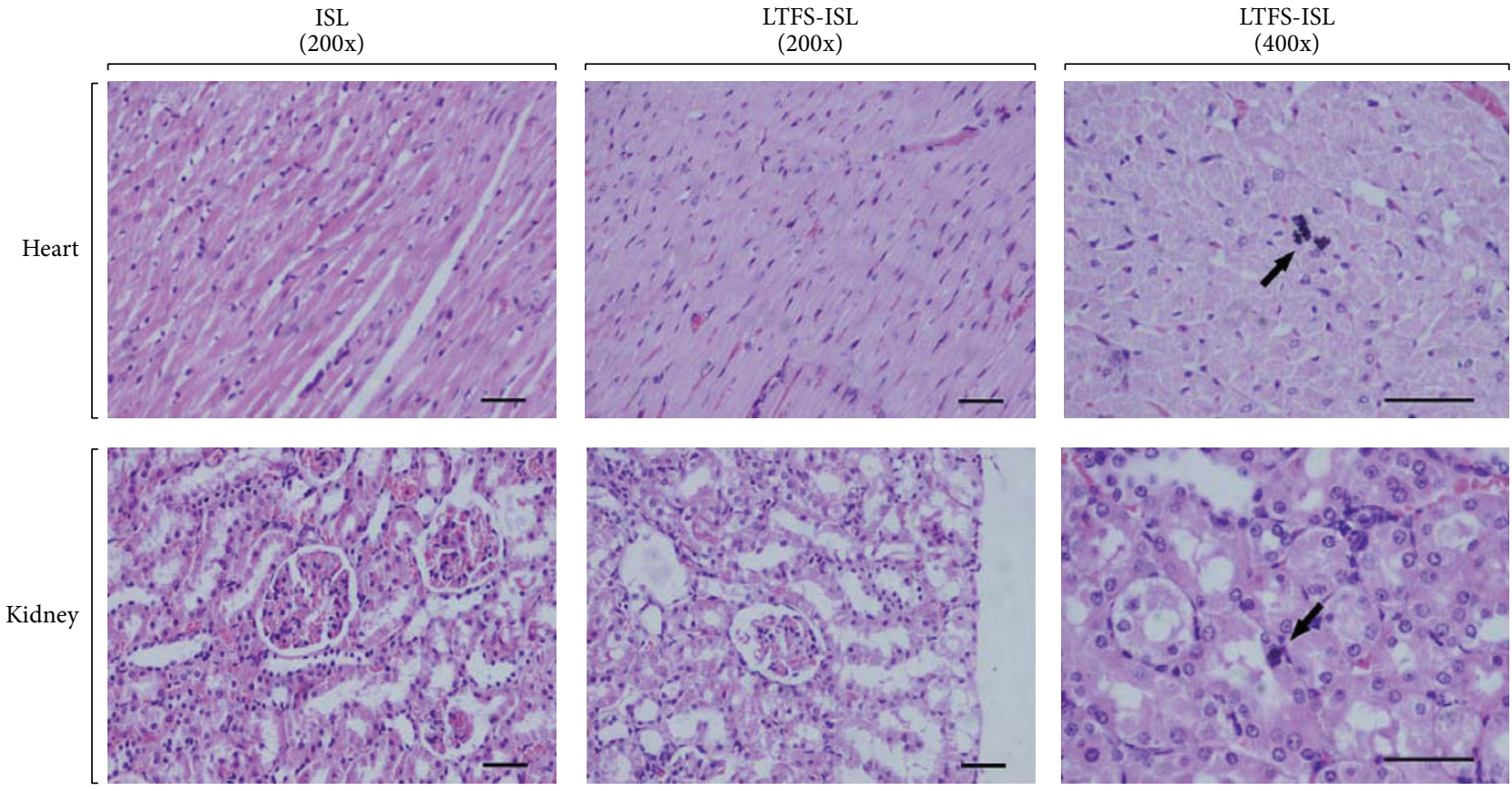

FIGURE 7: The effect of LTFS-ISL on the heart and kidneys. The arrows indicate that the carbon nanotubes remained in some organs. The scale bar is $100 \mu \mathrm{m}$.

\section{Conflict of Interests}

The authors declare that they have no conflict of interests.

\section{Acknowledgment}

This work was supported by the National Natural Science Foundation of China (nos. 30960515 and 81260487), the Doctor Foundation of Xinjiang Bingtuan (no. 2012BB020) and the Foundation from Shihezi University (ZRKXYB-13, YX07010, RCZX201115).

\section{References}

[1] T. Umemura, R. Kitaguchi, K. Inagaki, and H. Haraguchi, "Direct injection determination of theophylline and caffeine in blood serum by high-performance liquid chromatography using an ODS column coated with a zwitterionic bile acid derivative," Analyst, vol. 123, no. 8, pp. 1767-1770, 1998.

[2] H.-X. Liu, W.-H. Lin, X.-L. Wang, and J.-S. Yang, "Flavonoids from preparation of traditional Chinese medicines named SiniTang," Journal of Asian Natural Products Research, vol. 7, no. 2, pp. 139-143, 2005.

[3] M. Tawata, K. Aida, T. Noguchi et al., "Anti-platelet action of isoliquiritigenin, an aldose reductase inhibitor in licorice," European Journal of Pharmacology, vol. 212, no. 1, pp. 87-92, 1992.

[4] C. Zhan and J. Yang, "Protective effects of isoliquiritigenin in transient middle cerebral artery occlusion-induced focal cerebral ischemia in rats," Pharmacological Research, vol. 53, no. 3, pp. 303-309, 2006.

[5] J.-Y. Kim, S. J. Park, K.-J. Yun, Y.-W. Cho, H.-J. Park, and K.T. Lee, "Isoliquiritigenin isolated from the roots of Glycyrrhiza uralensis inhibits LPS-induced iNOS and COX-2 expression via the attenuation of NF- $\kappa \mathrm{B}$ in RAW 264.7 macrophages," European Journal of Pharmacology, vol. 584, no. 1, pp. 175-184, 2008.

[6] D.-C. Kim, S.-Y. Choi, S.-H. Kim et al., "Isoliquiritigenin selectively inhibits $\mathrm{H}_{2}$ histamine receptor signaling," Molecular Pharmacology, vol. 70, no. 2, pp. 493-500, 2006.

[7] Y. Sato, J.-X. He, H. Nagai, T. Tani, and T. Akao, "Isoliquiritigenin, one of the antispasmodic principles of Glycyrrhiza ularensis roots, acts in the lower part of intestine," Biological and Pharmaceutical Bulletin, vol. 30, no. 1, pp. 145-149, 2007.

[8] S. Tamir, M. Eizenberg, D. Somjen, S. Izrael, and J. Vaya, "Estrogen-like activity of glabrene and other constituents isolated from licorice root," Journal of Steroid Biochemistry and Molecular Biology, vol. 78, no. 3, pp. 291-298, 2001.

[9] B. Han, Q.-N. Li, S.-W. Wu, J.-G. Li, W. Chen, and W.-X. Li, "Selective adsorption of multi-walled carbon nanotubes with liquiritin and isoliquiritin," Yao Xue Xue Bao, vol. 42, no. 11, pp. 1222-1226, 2007.

[10] B. Han, W. Chen, W.-J. Jin, and S.-P. Liu, "Evaluation of AB-8 macroporous adsorption resin for adsorption of total flavones in liquorice," Nan Fang Yi Ke Da Xue Xue Bao, vol. 27, no. 3, pp. 265-267, 2007.

[11] B. Han, Q. -s. Zheng, W. Chen, X. Wang, Q. Wang, and L. $\mathrm{Li}$, "A novel process for extraction of isoliquiritigenin from licorice (Glycyrrhiza glabra) roots by facile convert," Chemistry of Nature Compounds, vol. 46, no. 4, pp. 523-527, 2010.

[12] D. Li, Z. Wang, H. Chen et al., "Isoliquiritigenin induces monocytic differentiation of HL-60 cells," Free Radical Biology and Medicine, vol. 46, no. 6, pp. 731-736, 2009.

[13] B. Han, W. Chen, Q. Zheng et al., "Determination of isoliquiritigenin and its distribution in mice by synchronous fluorescence spectrometry," Analytical Sciences, vol. 27, no. 11, pp. 1115-1119, 2011. 
[14] S. A. Hodge, M. K. Bayazit, K. S. Coleman, and M. S. Shaffer, "Unweaving the rainbow: a review of the relationship between single-walled carbon nanotube molecular structures and their chemical reactivity," Chemical Society Reviews, vol. 41, no. 12, pp. 4409-4429, 2012.

[15] H. Ali-Boucetta and K. Kostarelos, "Carbon nanotubes in medicine and biology: therapy and diagnostics," Advanced Drug Delivery Reviews, vol. 65, no. 15, pp. 1897-1898, 2013.

[16] Z. Liu, S. Tabakman, K. Welsher, and H. Dai, "Carbon nanotubes in biology and medicine: In vitro and in vivo detection, imaging and drug delivery," Nano Research, vol. 2, no. 2, pp. 85120, 2009.

[17] C.-W. Lam, J. T. James, R. McCluskey, and R. L. Hunter, "Pulmonary toxicity of single-wall carbon nanotubes in mice 7 and 90 days after intractracheal instillation," Toxicological Sciences, vol. 77, no. 1, pp. 126-134, 2004.

[18] J. C. Carrero-Sánchez, A. L. Elías, R. Mancilla et al., "Biocompatibility and toxicological studies of carbon nanotubes doped with nitrogen," Nano Letters, vol. 6, no. 8, pp. 1609-1616, 2006.

[19] K. Donaldson and C. A. Poland, "Nanotoxicology: new insights into nanotubes," Nature Nanotechnology, vol. 4, no. 11, pp. 708710, 2009.

[20] S. M. Ryan, G. Mantovani, X. Wang, D. M. Haddleton, and D. J. Brayden, "Advances in PEGylation of important biotech molecules: delivery aspects," Expert Opinion on Drug Delivery, vol. 5, no. 4, pp. 371-383, 2008.

[21] B. Zhao, H. Hu, A. Yu, D. Perea, and R. C. Haddon, "Synthesis and characterization of water soluble single-walled carbon nanotube graft copolymers," Journal of the American Chemical Society, vol. 127, no. 22, pp. 8197-8203, 2005.

[22] A. Sarafraz-Yazdi, A. Amiri, G. Rounaghi, and H. E. Hosseini, "A novel solid-phase microextraction using coated fiber based sol-gel technique using poly(ethylene glycol) grafted multi-walled carbon nanotubes for determination of benzene, toluene, ethylbenzene and o-xylene in water samples with gas chromatography-flam ionization detector," Journal of Chromatography A, vol. 1218, no. 34, pp. 5757-5764, 2011.

[23] B. Han, S. Ma, H. A. Aisa, X. C. Wang, and W. Chen, "Characterization andbiocompatibility of pegylated single walled carbon nanotubes," Chinese Journal of Hospital Pharmacy, vol. 32, no. 18, pp. 1415-1419, 2012.

[24] R. Zeineldin, M. Al-Haik, and L. G. Hudson, "Role of polyethylene glycol integrity in specific receptor targeting of carbon nanotubes to cancer cells," Nano Letters, vol. 9, no. 2, pp. 751757, 2009.

[25] R. Singh, D. Pantarotto, L. Lacerda et al., "Tissue biodistribution and blood clearance rates of intravenously administered carbon nanotube radiotracers," Proceedings of the National Academy of Sciences of the United States of America, vol. 103, no. 9, pp. 33573362, 2006.

[26] L. Lacerda, A. Soundararajan, R. Singh et al., "Dynamic imaging of functionalized multi-walled carbon nanotube systemic circulation and urinary excretion," Advanced Materials, vol. 20, no. 2, pp. 225-230, 2008. 

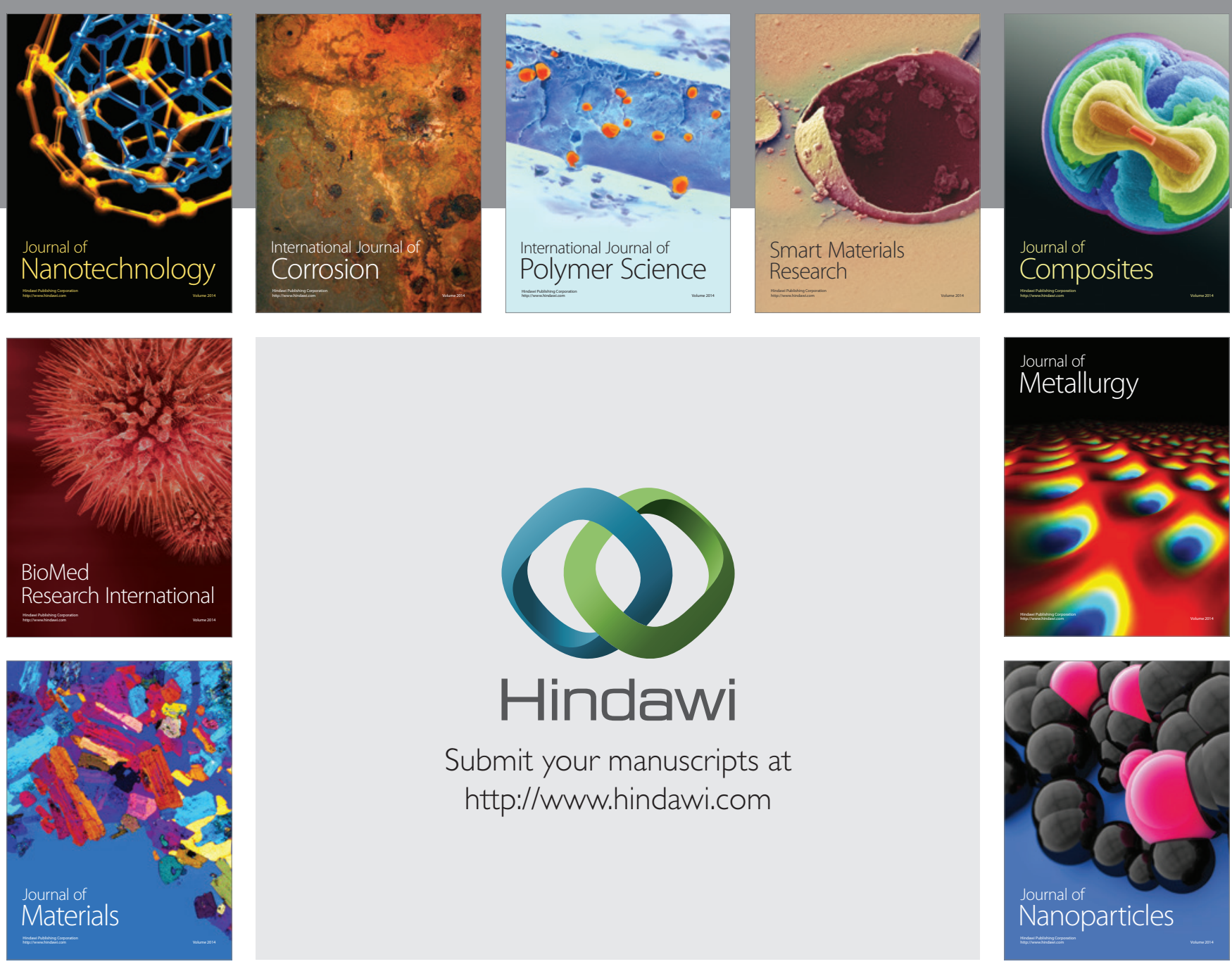

Submit your manuscripts at http://www.hindawi.com
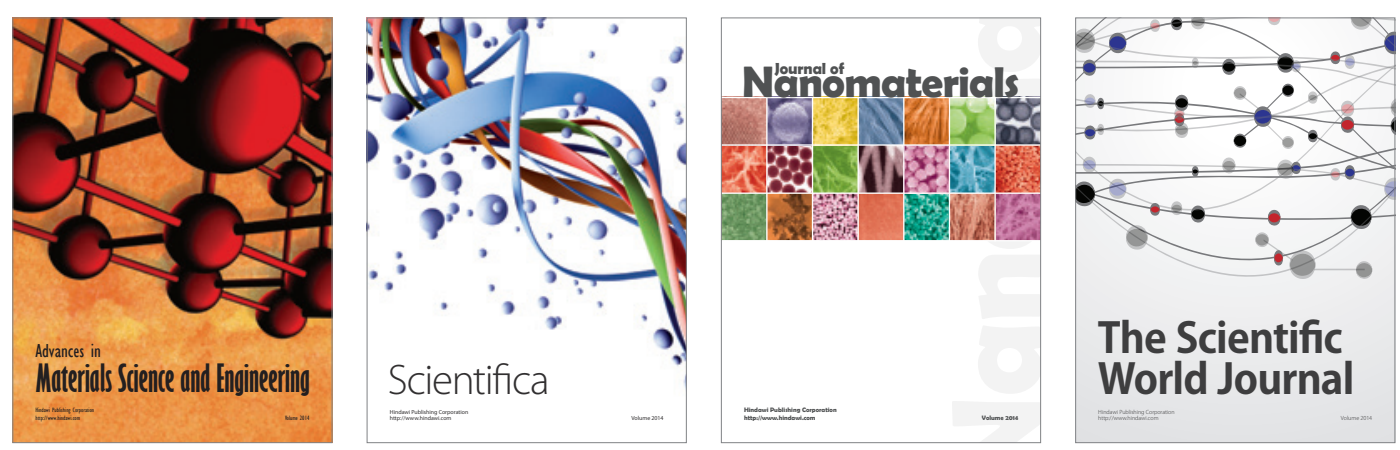

\section{The Scientific World Journal}
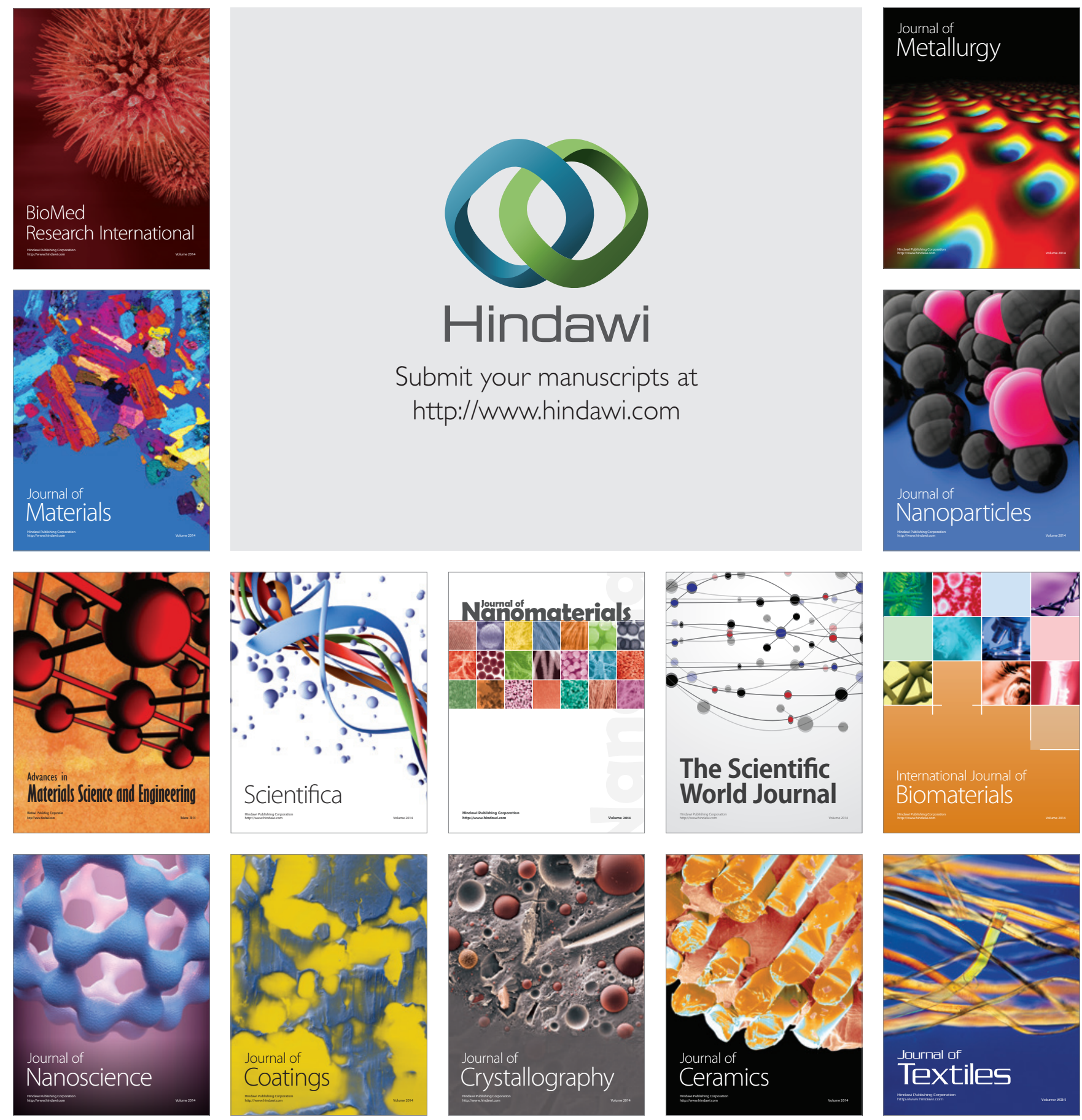